\title{
Service social
}

\section{Le travail insignifiant, par Denis Carrier, Montréal, Éditions Guérin, 1991, 183 pages.}

\section{Jeanne Bélanger}

Volume 40, numéro 2, 1991

Formation et évolution de la pratique en travail social

URI : https://id.erudit.org/iderudit/706537ar

DOI : https://doi.org/10.7202/706537ar

Aller au sommaire du numéro

Éditeur(s)

École de service social de l'Université Laval

ISSN

1708-1734 (numérique)

Découvrir la revue

Citer ce compte rendu

Bélanger, J. (1991). Compte rendu de [Le travail insignifiant, par Denis Carrier, Montréal, Éditions Guérin, 1991, 183 pages.] Service social, 40(2), 151-153.

https://doi.org/10.7202/706537ar d'utilisation que vous pouvez consulter en ligne.

https://apropos.erudit.org/fr/usagers/politique-dutilisation/ 
intéressant de parler de Monica McGoldrick, de Peggy Pann et de Hoffman. Ces auteurs nous amènent à réfléchir sur l'importance de la vision systémique d'aujourd'hui et de demain, surtout en ce qui concerne tout le principe du constructivisme et des différences des sexes en thérapie familiale. Cette limite en soi ne nous empêche pas de cerner les auteurs privilégiés par $M$. Lacroix et d'apprécier avec lui le concept et le modèle systémique. En service social, ce regard systémique n'est pas toujours compris étant donné que l'individu est toujours en première place. En ce sens, l'auteur contribue à nous ouvrir davantage de nouveaux horizons et à nous rendre plus sensibles aux nouvelles approches qui ont fait tout de même leurs preuves.

Encore une fois, c'est un texte qui demeure plutôt théorique malgré les nombreux cas cités en exemple. Les cliniciens vont surtout le feuilleter en raison de son contenu synthétique et non pas à cause de sa richesse sur le plan de l'intervention clinique. Ces mêmes cliniciens pourront s'en servir comme texte à lire et à consulter en formation personnelle. La théorie est bien vulgarisée et l'auteur réussit à garder le lecteur intéressé au sujet qu'il a choisi.

Pour conclure, c'est un volume qui a la qualité de parler des grandes lignes $\mathrm{d}^{\prime}$ orientation. C'est un livre facilement accessible qui peut introduire en service social la notion systémique, notion importante touchant l'individu et son environnement. On l'utilisera avec profit comme lecture de référence.

\section{Marie-Rose Parent \\ Centre hospitalier Pierre-Janet}

Hull

\section{Le travail insignifiant, par Denis CARRIER, Montréal, Éditions Guérin, 1991, 183 pages.}

Le travail insignifiant c'est l'avoir possible et le non-être assuré.

Le travail insignifiant c'est un travail qui peut nous rendre insignifiant. Nous pouvons nous donner un projet de société où l'économie sera davantage au service de la personne humaine et où chacun aura la possibilité de donner un sens à son travail et à sa vie.

Cette citation placée en exergue dans la première page de cet essai nous donne à penser que nous rencontrerons un allié avec qui nous pourrons "philosopher » sur le travail. D'ailleurs, à la fin du livre nous trouvons quelques pages blanches qui semblent attendre nos commentaires à la suite de notre lecture. Comme l'auteur de ce livre, Denis Carrier, a œuvré dans le domaine du développement communautaire et dans celui de la santé-sécurité au travail, peut-être nous racontera-t-il quelques-unes de ses expériences?

L'auteur veut nous démontrer que le travail tel qu'il se présente actuellement risque souvent de devenir insignifiant. Pour ce faire, il traite les thèmes de l'économie, de l'environnement et des droits sociaux, sous l'angle des statistiques.

Dans le premier chapitre portant sur l'économie, l'auteur aborde le problème de la précarité des emplois : "Une étude du Conseil économique du Canada nous apprend que depuis 1975 le travail à temps partiel au Québec est intervenu dans $40 \%$ des nouveaux emplois $»(p .25)$. II parle également du sousemploi (comme le diplômé d'université qui se retrouve chauffeur de taxi). II traite aussi du chômage, du libre-échange et des effets de la TPS (taxe peu 
sympathique !) qui pourraient contribuer à une augmentation du travail au noir. Il commente enfin le travail à rentabilité différée, tel celui de la femme au foyer.

Deuxième grand thème abordé : l'environnement. Sous le premier soustitre "Penser globalement et agir localement ", nous trouvons des chiffres qui ne sont pas très flatteurs pour les entreprises : "Sur 2900 compagnies et institutions publiques observées, $2 \%$ des investissements $d^{\prime}$ immobilisation, en moyenne, sont consacrés à la réduction de la pollution et à l'économie de l'énergie" (p. 39). II parle également des pluies acides, de l'effet de serre, des répercussions de la pollution sur la santé (asthme, cancer) et finalement de la pollution spirituelle dans le sens où « les relations humaines ont été polluées par un souci de pouvoir et de domination " (p. 53).

Dans le troisième chapitre intitulé "La personne au travail ", l'auteur traite des problèmes rencontrés chez les jeunes (suicide, chômage et violence), chez les femmes (l'inégalité salariale, les congés parentaux) et chez les aînés (pression exercée sur eux afin qu'ils prennent une préretraite non désirée).

Quant au quatrième chapitre "La personne avant toute chose ", différents thèmes y sont abordés en vrac, tels la productivité à la suédoise, l'absentéisme, les cadres, la formation, I'action volontaire, l'adhocratie et la gestion participante. Ces deux derniers thèmes auraient eu avantage à être plus approfondis, car ces approches semblent offrir des éléments de solution intéressants pour une société plus équitable.

Sous le thème "Les droits sociaux", l'auteur parle d'un projet social québécois qu'il développe davantage au chapitre suivant sous le titre " $\mathrm{Se}$ mettre au monde " : il définit alors la société sociale, économique et politique qu'il souhaiterait pour le Québec. Il croit en effet que " c'est en bonne partie au sein de l'entreprise que le Québec international va se mettre au monde. Le Québec s'est donné une Charte des droits et libertés de la personne qui est qualifiée de modèle et d'innovatrice sur le plan du droit international. Nous pourrions participer activement au développement d'un droit international nouveau en proposant une " charte de la personne au travail » (p. 152).

L'auteur a voulu, semble-t-il, mettre en évidence la difficulté pour l'individu de travailler dans un contexte économique qui ne favorise pas le développement de son potentiel à cause des facteurs tels le "bumpage ", le travail à temps partiel, le travail en milieu trop compétitif, etc. De fait, le travail effectué dans de telles conditions perd de son sens. De plus, les milieux de travail ne respectent pas toujours les valeurs personnelles de l'individu avec le résultat que celui-ci se sent dans un état de dualité intérieure entre ses aspirations profondes et la réalité du travail. C'est ce malaise qu'a tenté d'analyser l'auteur au moyen des statistiques, mais les chiffres à eux seuls ne sont pas suffisamment convaincants.

En effet, la faiblesse d'analyse gêne cet auteur lorsque vient le temps de faire des propositions concrètes, telle l'élaboration d'un véritable projet social; ses propositions demeurent trop vagues pour être réalistes à court et à long terme. De plus, il est difficile de faire une synthèse des différents chapitres parce qu'ils ne présentent souvent qu'une suite de données parfois sans liens apparents entre elles. II est dommage que les citations philosophiques contenues dans le livre, comme celles de Hubert Reeves et de Frédérick Back, ne réussissent pas à prendre leur place à travers la prédominance des statistiques, faute d'être soutenue par une pensée mieux articulée.

Les chapitres "La personne au travail » et "La personne avant toute chose " auraient dû constituer les deux premiers chapitres auxquels les thèmes de l'économie, de l'environnement et des droits sociaux auraient pu se greffer. 
En outre, une présentation plus méthodique aurait grandement contribué à rehausser le contenu de ce livre. Quant au titre, il ne correspond pas suffisamment au contenu : l'auteur ne réussit pas réellement à analyser l'insignifiance du travail et le lecteur demeure submergé par les chiffres.

Le nombre de références toutefois est considérable : 405 notices bibliographiques qui ont été puisées principalement dans des journaux (La Presse, Le Devoir, Le Soleil, Le Journal de Montréal), des revues (L'actualité, Écologie) ainsi que des documents gouvernementaux. II est à noter que si les références avaient été données en ordre alphabétique, il aurait été plus facile de repérer les auteurs des articles, mais c'est tout de même un travail de recherche imposant et le plus grand mérite de ce livre.

Bref, le praticien qui recherche une réflexion personnelle sur le travail risque fort de trouver indigeste l'amoncellement de statistiques contenues dans cet essai. Par contre, le lecteur qui cherche des statistiques brèves sur les différents aspects du travail (politique, économique et social) a de fortes chances d'être satisfait. Ce livre pourrait avoir sa place dans la bibliothèque des professeurs et des étudiants en sciences humaines qui ont à analyser les différents problèmes sociaux liés au travail. De plus, le coût du livre est abordable.

Jeanne Bélanger

Andragogue

Hull

Théorie et pratiques en organisation communautaire, sous la direction de Laval DouCET et Louis FAVREAU, Sillery, Presses de l'Université du Québec, 1991, 468 pages.

Au cours des années 60 , les textes dont les étudiants québécois en organisation communautaire disposaient étaient la plupart du temps le fait d'auteurs américains : rarissimes étaient les travaux pertinents écrits en français. Nous plaquions donc les théories et pratiques de nos voisins du sud à la réalité qui était la nôtre, mais avec les discordances faciles à prévoir : il n'y a pas homologie des pratiques entre une société plutôt individualiste et une autre " tricotée plus serré ". La société québécoise disposait pourtant d'un important bagage d'expériences d'organisation communautaire, mais nous faisions comme si nous ne pouvions encore en tirer des leçons comme les Américains le faisaient pourtant pour eux-mêmes.

Vinrent ensuite les années 70 au cours desquelles un fort vent de dogmatisme souffla sur l'organisation communautaire; plusieurs n'avaient plus que faire de ces recettes empiriquement distillées par l'expérience et se sentaient davantage attirés par la théorie pure. L'influence américaine fut remplacée par celle du léninisme, du stalinisme, du maoïsme, et autres Enver Hoxha. II s'agissait donc de suivre la ligne juste, souvent la plus droite mais pas nécessairement la plus praticable.

II a fallu attendre les années 80 pour voir paraître les premiers livres d'organisation communautaire, mais le défi n'était pas facile à relever : il fallait relire l'expérience américaine avec laquelle nous partageons la sensibilité et une expérience semblables, mais en l'appliquant à notre contexte. Voilà la tâche à laquelle se sont attaqués avec bonheur les collègues Doucet et Favreau dans ce qui constitue le premier vrai livre québécois d'introduction à l'organisation communautaire. 